Noname manuscript No.

(will be inserted by the editor)

\title{
Coxeter Groups and Hyperbolic Manifolds
}

\author{
Brent Everitt ${ }^{\star}$ \\ Department of Mathematics, University of York, York YO10 5DD, England
}

The date of receipt and acceptance will be inserted by the editor

\section{Introduction}

In the last quarter of a century, 3-manifold topology has been revolutionized by Thurston and his school. This has generated a huge literature on hyperbolic 3-manifolds, building on the classical body of knowledge already existing in 2-dimensions. Balanced against this is a relative paucity of techniques and examples of hyperbolic $n$-manifolds for $n \geq 4$. Recent work of Ratcliffe and Tschantz has provided examples of smallest possible volume when $n=4$ by identifying the faces of polytopes (23], see [10] for a seminal example). On the other hand, in a construction that is more "algebraic" in nature, they computed in [25] the covolumes of the principle congruence subgroups of level $p$ a prime in $P O_{1, n} \mathbb{Z}$, thus giving an infinite family of manifolds, with known volume, in every dimension. In a similar vein, [12] allows one to construct manifolds in arbitrary dimensions by considering the kernels of representations of Coxeter groups onto finite classical groups. However, it turns out that the volumes of the manifolds arising from both constructions are not particularly small (eg: in [25] the smallest 6-dimensional example has Euler characteristic $|\chi|=44226)$. Thus there seem to be no constructions in arbitrary dimensions that give examples of very small volume.

This paper is an attempt to redress this. A construction is given that essentially makes algebraic the classical geometric idea of identifying the faces of a polytope. Algebraic is meant in the following sense. Any finite volume hyperbolic manifold arises as a quotient $\mathbb{H}^{n} / \Pi$ of $\mathbb{H}^{n}$ by the action of a group $\Pi$ acting properly, freely, cofinitely by isometries. The fundamental group of the manifold is thus isomorphic to $\Pi$. Moreover, by Mostow rigidity [19, 20, 22], finite volume hyperbolic $n$-manifolds, for $n \geq 3$, are completely determined by their fundamental groups, in that two such manifolds are isometric precisely when their fundamental groups are isomorphic. Thus, to construct $M=\mathbb{H}^{n} / \Pi$, we construct $\Pi$.

The geometric notions of a free proper cofinite action can be replaced by the algebraic conditions that $\Pi$ is a torsion free lattice in the Lie group Isom $\mathbb{H}^{n}$. The task can then be spilt into

\footnotetext{
* e-mail: bje1@york.ac.uk
} 
two parts. Start with a Coxeter group $\Gamma$ embedded as a lattice in Isom $\mathbb{H}^{n}$. Fortunately, there already exists an extensive literature on such hyperbolic Coxeter groups (see [30] and the references there). By Selbergs lemma [26], such a $\Gamma$ is virtually torsion free (as indeed is any abstract Coxeter group), so the second part is to find the desired $\Pi$ as a subgroup of finite index. It is the machinery necessary for this second step that preoccupies most of this paper-it is combinatorial in nature and uses only the algebraic properties of $\Gamma$. Basic properties of the resulting manifolds, such as volume and orientability, can then be determined from the structure of $\Gamma$.

The paper is organised as follows: $\S 2$ contains preliminary material about Coxeter groups, in particular the crucial results of Vinberg that ensure an abstract Coxeter group can be embedded as a lattice in Isom $\mathbb{H}^{n} ; \S 3$ gives the construction and the important role played by the conjugacy classes of finite Coxeter groups; these classes are determined in $\S 4$, where the results of Roger Carter's lengthy investigation [6] are summarized in a form amenable to our purposes; computing volumes is achieved via the techniques of $\S 5$ and $\S 6$ contains three examples, one in each of 4,5 and 6 -dimensions.

It is a pleasure to acknowledge many helpful discussions with Colin Maclachlan and Patrick Dorey.

\section{Coxeter Groups}

Let $\Gamma$ be a group and $S \subset \Gamma$ finite. The pair $(\Gamma, S)$ is called a Coxeter system (and $\Gamma$ a Coxeter group) if $\Gamma$ admits a presentation with generators $s_{\alpha} \in S$, and relations,

$$
\left(s_{\alpha} s_{\beta}\right)^{m_{\alpha \beta}}=1 \text {, }
$$

where $m_{\alpha \beta} \in \mathbb{Z}^{+} \cup\{\infty\}$ and $m_{\alpha \alpha}=1$ (see [1, 17] for basic facts about Coxeter groups). It is customary to omit relations for which $m_{\alpha \beta}=\infty$. Associated to any Coxeter group is its symbol, with nodes indexed by the $s_{\alpha}$, where nodes $s_{\alpha}$ and $s_{\beta}$ are joined by an edge labelled $m_{\alpha \beta}$ if $m_{\alpha \beta} \geq 4$, an unlabelled edge if $m_{\alpha \beta}=3$ and a dotted edge if $m_{\alpha \beta}=\infty$. It is a standard abuse of notation to denote a Coxeter group and its symbol by the same letter.

Coxeter groups satisfy a Freiheitssatz, in the sense that for any $S^{\prime} \subseteq S$, the subgroup of $\Gamma$ generated by the $s_{\alpha} \in S^{\prime}$ is also a Coxeter group with symbol obtained from $\Gamma$ by removing those nodes not in $S^{\prime}$ and their incident edges. In general, a subsymbol of $\Gamma$ is some subset of the vertices and their incident edges. If a symbol $\Gamma$ is disconnected with connected components $\Gamma_{1}, \ldots, \Gamma_{k}$, then $\Gamma$ is isomorphic to $\Gamma_{1} \times \cdots \times \Gamma_{k}$. A group with connected symbol is called irreducible.

It should come as no surprise that in finding torsion free subgroups of Coxeter groups the finite, or spherical, Coxeter groups play a central role. The finite irreducible Coxeter groups are well known to be: the Weyl groups of simple Lie algebras over $\mathbb{C}$, the dihedral groups, the group of symmetries of a regular dodecahedron, and the group of symmetries of the regular 4-dimensional polytope, the 120-cell. The Killing-Cartan notation will be used throughout this paper, in which the Weyl groups fall into three infinite classical families,

$$
A_{n}(n \geq 1), B_{n}(n \geq 2) \text { and } D_{n}(n \geq 4),
$$

as well as five exceptional groups, $G_{2}, F_{4}, E_{6}, E_{7}$ and $E_{8}$. The non-Weyl groups are the dihedral $I_{2}(m)$ for $m \geq 3$, and the dodecahedron and 120-cell symmetry groups $H_{3}$ and $H_{4}$ (note that there are the exceptional isomorphisms $A_{2} \cong I_{2}(3), B_{2} \cong I_{2}(4)$ and $G_{2} \cong I_{2}(6)$ ). The symbols 
for the Weyl groups are in Tables 1-2, and the non-Weyl groups in Proposition 3 and Table 3 . Given an arbitrary (not necessarily connected) Coxeter symbol, it represents a finite Coxeter group if and only if each of its connected components is in the list above.

A slightly smaller role is reserved for the parabolic Coxeter (or affine Weyl) groups: the irreducible ones are $\tilde{A}_{n}(n \geq 1), \tilde{B}_{n}(n \geq 3), \tilde{C}_{n}(n \geq 2), \tilde{D}_{n}(n \geq 4), \tilde{E}_{6}, \tilde{E}_{7}, \tilde{E}_{8}, \tilde{F}_{4}$, and $\tilde{G}_{2}$. For their symbols, consult [17, §2.5]. In general, a Coxeter group is parabolic (or Euclidean) if and only if its symbol has connected components corresponding to the groups in this list.

A convex polytope $P$ in $\mathbb{H}^{n}$ is an intersection,

$$
P=\bigcap_{s \in S} H_{s}^{-},
$$

of closed half spaces $H_{s}^{-}$bounded by hyperplanes $H_{s}$. We will assume that $S$ is finite (although we could take locally finite), and to avoid degenerate cases, that $P$ contains a non-empty open subset of $\mathbb{H}^{n}$. A Coxeter system $(\Gamma, S)$ is hyperbolic of dimension $n$ if and only if there is a $P \subset$ $\mathbb{H}^{n}$ such that assigning $s_{\alpha} \in S$ to the reflection in the hyperplane $H_{s_{\alpha}}$ induces an isomorphism between $\Gamma$ and the group generated by reflections in the faces of $P$. Call $\Gamma$ cocompact (resp. cofinite) if $P$ is compact (resp. of finite volume) in $\mathbb{H}^{n}$.

Given an abstract Coxeter system $(\Gamma, S)$, when can one realise it as a cocompact/cofinite hyperbolic Coxeter group? Dealing first with hyperbolicity, a Gram matrix $G(\Gamma)$ for $\Gamma$ has rows and columns indexed by $S$; if $m_{\alpha \beta} \in \mathbb{Z}^{+}$, set the $\alpha \beta$-th and $\beta \alpha$-th entries of $G(\Gamma)$ to be $-\cos \left(\pi / m_{\alpha \beta}\right)$, and if $m_{\alpha \beta}=\infty$, choose the $\alpha \beta$-th and $\beta \alpha$-th entries to be some real $c_{\alpha \beta}$ with $c_{\alpha \beta} \leq-1$. Recall that a symmetric matrix has signature $(p, q)$ if it has precisely $p$ eigenvalues that are $<0$ and $q$ that are $>0$.

Theorem 1 ([30], Theorem 2.1) A Coxeter group is n-dimensional hyperbolic if there exist $c_{\alpha \beta}$ 's such that $G(\Gamma)$ has signature $(1, n)$.

The condition is not necessary: the reflection subgroup of the group of automorphisms of a Lorentzian lattice over $\mathbb{Z}[(\sqrt{5}+1) / 2]$ is a 6 -dimensional hyperbolic Coxeter group with Gram matrix having signature $(2,4)$ [ [ 7 . Note that in [17, §6.8-6.9], the sufficient condition of Theorem 1 (as well as the condition that the Gram matrix be non-degenerate) is taken as the definition of hyperbolic Coxeter group, and so a more restricted class of groups is obtained, indeed, classified. A classification in the general case still eludes us.

The configuration of the bounding hyperplanes of $P$ can be described as follows. If $m_{\alpha \beta} \in$ $\mathbb{Z}^{+}$then the corresponding hyperplanes intersect with dihedral angle $\pi / m_{\alpha \beta}$; if $c_{\alpha \beta}=-1$ they are parallel, that is, intersect at infinity; and if $c_{\alpha \beta}<-1$ they are ultraparallel (they do not intersect in the closure of $\mathbb{H}^{n}$ ) with a common perpendicular geodesic of length $\eta_{\alpha \beta}$, where $-\cosh \eta_{\alpha \beta}=c_{\alpha \beta}$. Because of these last two, some authors prefer to embellish the Coxeter symbol, replacing the dotted edges by thick solid ones if $c_{\alpha \beta}=-1$, or by ones labelled $\eta_{\alpha \beta}$.

To determine if $(\Gamma, S)$ is realised by a cocompact/cofinite $P \subset \mathbb{H}^{n}$, let $\mathscr{F}=\mathscr{F}(\Gamma, S)$ be the collection of finite subgroups of $\Gamma$ generated by subsets $S^{\prime} \subset S$ (including $S^{\prime}=\emptyset$, which generates the trivial group). Partially order $\mathscr{F}$ by inclusion. Similarly $\overline{\mathscr{F}}$ is the poset obtained by taking both the finite and the parabolic subgroups. The poset of an abstract combinatorial polytope $P$ is the set of cells of $P$, partially ordered by inclusion.

Theorem 2 ([30], Proposition 4.2) An n-dimensional hyperbolic Coxeter group $(\Gamma, S)$ is cocompact (resp. cofinite) iff $\mathscr{F}$ (resp. $\overline{\mathscr{F}}$ ) is isomorphic as a partially ordered set to the poset of some n-dimensional abstract combinatorial polytope. 
A slight variation yields a very useful $\Gamma$-complex. Let $\Gamma \mathscr{F}=\Gamma \mathscr{F}(\Gamma, S)$ be the set of all right cosets of the subgroups in $\mathscr{F}$. Partially order this set by inclusion and let $\Sigma$ be the affine complex realising $\Gamma \mathscr{F}$ : the $k$-cells of $\Sigma$ are the chains $\sigma_{0}<\sigma_{1}<\cdots<\sigma_{k}$ with $\sigma_{i} \in \Gamma \mathscr{F}$. The group $\Gamma$ acts cellularly on $\Sigma$ by right multiplication. If $\Gamma$ is hyperbolic then $\Sigma$ is just the barycentric subdivision of the tessellation of $\mathbb{H}^{n}$ by congruent copies of the polytope $P$. But in fact for any Coxeter system, the complex turns out to be negatively curved:

Theorem 3 Let $(\Gamma, S)$ be a Coxeter system and equip $\Sigma$ with the natural piecewise Euclidean metric where each $k$-cell is isometric to a regular $k$-dimensional Euclidean simplex. Then this is a CAT $(0)$ space with the $\Gamma$ action by right multiplication isometric.

For basic facts concerning $\operatorname{CAT}(0)$ spaces, [9] is recommended, where this result is Corollary 6.7.5 (see also [21).

\section{Torsion free subgroups of Coxeter groups}

The construction of torsion free subgroups of a Coxeter group requires answers to the following two questions: where exactly is the torsion, and given a subgroup, when does it avoid it?

Theorem 4 Let $(\Gamma, S)$ be a Coxeter system. Then any element of finite order in $\Gamma$ is conjugate to an element of a finite subgroup generated by some $S^{\prime} \subseteq S$.

This is an exercise in Bourbaki [1], and an algebraic proof using root systems appears in [5]. We provide a geometrical proof that is presumably well known, but does not appear to be in the literature:

Proof Consider the isometric $\Gamma$ action by right multiplication on the complex $\Sigma$ above. Any torsion element $\gamma$ in a group of isometries of a $\operatorname{CAT}(0)$ space has a fixed point 调, Corollary II.2.8], and since the $\Gamma$ action is cellular, $\gamma$ must fix a $k$-cell $\sigma_{0}<\sigma_{1}<\cdots \sigma_{k}$ with $\sigma_{i}=\left\langle S_{i}\right\rangle g$ for $g \in \Gamma$ and $S_{i} \subset S$ with $\left\langle S_{i}\right\rangle$ finite. Thus, $g \gamma g^{-1}$ fixes the $k$-cell $\left\langle S_{0}\right\rangle<\cdots<\left\langle S_{k}\right\rangle$.

To address the second question above, it will turn out to be convenient to use the language of permutation modules rather than subgroups. Let $\Omega$ be a finite set and $U$ a vector space over $\mathbb{C}$ with basis $\Omega$. An action of any group $\Gamma$ on $\Omega$ can be extended linearly to an action on $U$, thus giving $U$ the structure of a (permutation) $\mathbb{C} \Gamma$-module. Call the module transitive if it arises via a transitive $\Gamma$-action on $\Omega$.

Conjugacy classes of subgroups $\Pi$ of index $|\Omega|$ in a group $\Gamma$ are in one to one correspondence with equivalence classes of transitive representations $\Gamma \rightarrow \operatorname{Sym} \Omega$. These in turn are in one to one correspondence with isomorphism classes of transitive permutation $\mathbb{C} \Gamma$-modules.

Let $\mathscr{C}$ be a conjugacy class of torsion elements in $\Gamma$. We will say that the $\mathbb{C} \Gamma$-module $U$ avoids $\mathscr{C}$ when the corresponding conjugacy class of subgroups has empty intersection with $\mathscr{C}$; $U$ is torsion free when it avoids the conjugacy classes of all torsion elements. The following is well known and easily proved:

Proposition 1 Let $U$ be a $\mathbb{C} \Gamma$-module for some group $\Gamma$ with basis $\Omega$. Then $U$ is torsion free exactly when it avoids the conjugacy classes of elements of prime order. Moreover, if $\mathscr{C}$ is such a class, and $\gamma \in \mathscr{C}$ any element, then $U$ avoids $\mathscr{C}$ exactly when $\gamma$ fixes no point of $\Omega$. 
So, if we had a list of representatives for the conjugacy classes of prime order torsion in $\Gamma$, verifying that a given $U$ is torsion free would become the simple matter of checking that no element in the list fixed a point of $\Omega$. By Theorem $\sharp$, such a list could be compiled for in particular for $(\Gamma, S)$ a Coxeter group by listing representatives of the conjugacy classes in the finite subgroups generated by $S^{\prime} \subseteq S$. Indeed, we can restrict to the finite subgroups generated by $S^{\prime}$ that are maximal with the property that they generate a finite subgroup. The list so obtained will very probably include redundancies, but will certainly be complete, which is clearly all that matters for the task at hand. A discussion of conjugacy in finite Coxeter groups is the subject of $\S 4$.

Rather than dealing with an indigestible whole, we can divide the task of finding a torsion free $U$ into manageable pieces. Given $\mathbb{C} \Gamma$-modules $U_{i}, i=1,2$, with bases $\Omega_{i}$, let $\Gamma$ act on $\Omega_{1} \times \Omega_{2}$ via $(\mathbf{u}, \mathbf{v})^{\gamma}=\left(\mathbf{u}^{\gamma}, \mathbf{v}^{\gamma}\right)$. Extending this action linearly to the complex vector space with basis $\Omega_{1} \times \Omega_{2}$ gives a permutation $\mathbb{C} \Gamma$-module that may be identified with $U_{1} \otimes U_{2}$. The problem is that $U_{1} \otimes U_{2}$ may not be transitive. Choose an orbit in the $\Gamma$-action on $\Omega_{1} \times \Omega_{2}$, and extend linearly to a $\Gamma$-action on the $\mathbb{C}$-vector space with basis this orbit. By an abuse of notation, we will use $U_{1} \otimes U_{2}$ to denote any one of the resulting (transitive permutation) $\mathbb{C} \Gamma$-modules.

The following follows by definition and Proposition 1

Lemma 1 Let $U_{i}, i=1,2$, be $\mathbb{C} \Gamma$-modules and $\mathscr{C}$ a conjugacy class of torsion elements in the group $\Gamma$. Then $U_{1} \otimes U_{2}$ avoids $\mathscr{C}$ if and only if at least one of the $U_{i}$ does.

Thus we may build a torsion free $\mathbb{C} \Gamma$-module $U$ by finding $U_{1}, \ldots, U_{k}$ that collectively avoid all the conjugacy classes of torsion elements (that is, each such class is avoided by at least one of the $U_{i}$ ), and then letting $U=\otimes U_{i}$. The index in $\Gamma$ of the corresponding subgroups $\Pi$ will be at most $\prod\left|\Omega_{i}\right|$, where $\Omega_{i}$ is the basis for $U_{i}$. On the other hand, if $\mathscr{L}(\Gamma)$ is the lowest common multiple of the orders of elements of $\mathscr{F}(\Gamma)$, then any torsion free subgroup of $\Gamma$ has index equal to a positive integer multiple of $\mathscr{L}(\Gamma)$. This gives bounds on the index. It is worth noting that the minimal index of a torsion free subgroup need not be $\mathscr{L}(\Gamma)$; in fact there are examples in 3 -dimensions where the minimal index can be any positive multiple of $\mathscr{L}(\Gamma)$ (see [11, 18]).

\section{Conjugacy in finite Coxeter groups}

We saw in $\S 3$ that a list of representatives for the conjugacy classes of torsion elements in $(\Gamma, S)$ could be obtained by listing the conjugacy class representatives of the finite subgroups generated by maximal $S^{\prime} \subseteq S$. If any such finite subgroup is reducible, then its conjugacy classes are easily obtained from those of its irreducible components. Thus, we need only consider conjugacy in irreducible finite Coxeter groups.

There are then two possibilities to consider: the Weyl groups and the non-Weyl groups, and these are dealt with separately. Many of the Weyl groups have alternative descriptions in terms of well-known finite groups. For example, those of type $A_{n}$ are isomorphic to the symmetric groups $\mathfrak{S}_{n+1}$; those of type $B_{n}$ arise as the split extension

$$
1 \longrightarrow \bigoplus^{n} \mathbb{Z}_{2} \longrightarrow B_{n} \longrightarrow \mathfrak{S}_{n} \longrightarrow 1,
$$

where for $\sigma \in \mathfrak{S}_{n}$ and $x_{k}$ the $\mathbb{Z}_{2}$-tuple with 1 in the $k$-th position and zeroes elsewhere, we have $\sigma^{-1} x_{k} \sigma=x_{\sigma(k)}$ (the group of so-called signed permutations). Similarly for those of type 
$D_{n}$ but with $\mathbb{Z}_{2}^{n}$ replaced by $\mathbb{Z}_{2}^{n-1}$ (the even signed permutations). Thus their conjugacy classes can be determined on a case by case basis. Alternatively, [6] determines them conceptually using their structure as reflection groups, and it is the results from here that we use.

\subsection{The Weyl groups}

The description is couched in terms of root systems (see [17. Chapters 1 and 2]). Let $V$ be a Euclidean space with orthogonal basis $\left\{\mathbf{e}_{1}, \ldots, \mathbf{e}_{n}\right\}$ and positive definite bilinear form defined by $\left\langle\mathbf{e}_{i}, \mathbf{e}_{j}\right\rangle=\delta_{i j}$, the Kronecker delta. For any $\mathbf{v} \in V$, let $s_{\mathbf{v}}$ be the linear reflection in the hyperplane orthogonal to $\mathbf{v}$. Tables 1 - 2 give the standard representations of the Weyl groups acting on $V$ : the subgroup of the orthogonal group $O(V)$ generated by the $s_{\mathbf{v}}$ where the $\mathbf{v}$ label the nodes of the symbol, is isomorphic to the abstract Coxeter group having that symbol. These $\mathbf{v}$ are a set of simple roots.

If $(\Gamma, S)$ is an irreducible Weyl group, identify its symbol with the appropriate one in Tables 112, thus identifying $S$ with the $s_{\mathbf{v}}$. It turns out [6] that the conjugacy classes in $\Gamma$ correspond to certain diagrams, closely related to the Coxeter symbol. We now proceed to give the diagrams, and, with $\S 3$ in mind, a method for obtaining a representative for the conjugacy class in terms of the generating reflections for $\Gamma$.

The diagrams for each group are given in Theorems 5 - 8 below. For each one, label the nodes (if they do not come prelabelled) by roots from the appropriate root system in Tables 1 2 , in such a way that if the nodes labelled $\mathbf{u}$ and $\mathbf{v}$ are connected by an edge labelled $m$, then

$$
\frac{4\langle\mathbf{v}, \mathbf{u}\rangle\langle\mathbf{u}, \mathbf{v}\rangle}{\langle\mathbf{v}, \mathbf{v}\rangle\langle\mathbf{u}, \mathbf{u}\rangle}=m-2 .
$$

In fact, we always have $m=2$ or 3 , and the diagrams use the same labelling conventions as for Coxeter symbols. If the diagram can be identified with a subsymbol of the Coxeter symbol, then a labelling is easily obtained-take a labelling by simple roots off the Coxeter symbol. Otherwise, it can be harder, as in the example below.

Colour the nodes of the labelled diagram black and white with a node of one colour joined only to nodes of the other. Let $\mathscr{B}$ and $\mathscr{W}$ be the sets of nodes of the two colours. Then a representative of the conjugacy class corresponding to the diagram is given by,

$$
\prod_{\mathbf{v} \in \mathscr{B}} s_{\mathbf{v}} \prod_{\mathbf{u} \in \mathscr{W}} s_{\mathbf{u}}
$$

We use the algebraists convention of reading such expressions from left to right. If $\mathbf{v}$ isn't a simple root, then $s_{\mathbf{v}}$ is not identified with one of the generating reflections of $\Gamma$. In this case, find a simple $\mathbf{v}^{\prime}$ and an element $w \in \Gamma$, given in terms of the generators, such that the image of $\mathbf{v}$ under $w$ is $\mathbf{v}^{\prime}$. Then $s_{\mathbf{v}}=w s_{\mathbf{v}^{\prime}} w^{-1}$, an expression in terms of the generating reflections.

For example, suppose we have a group of type $E_{6}$ with generators,

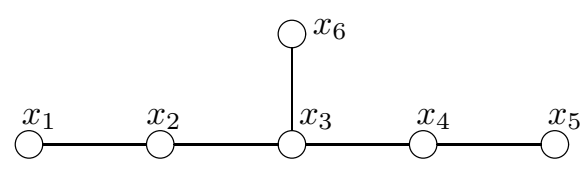




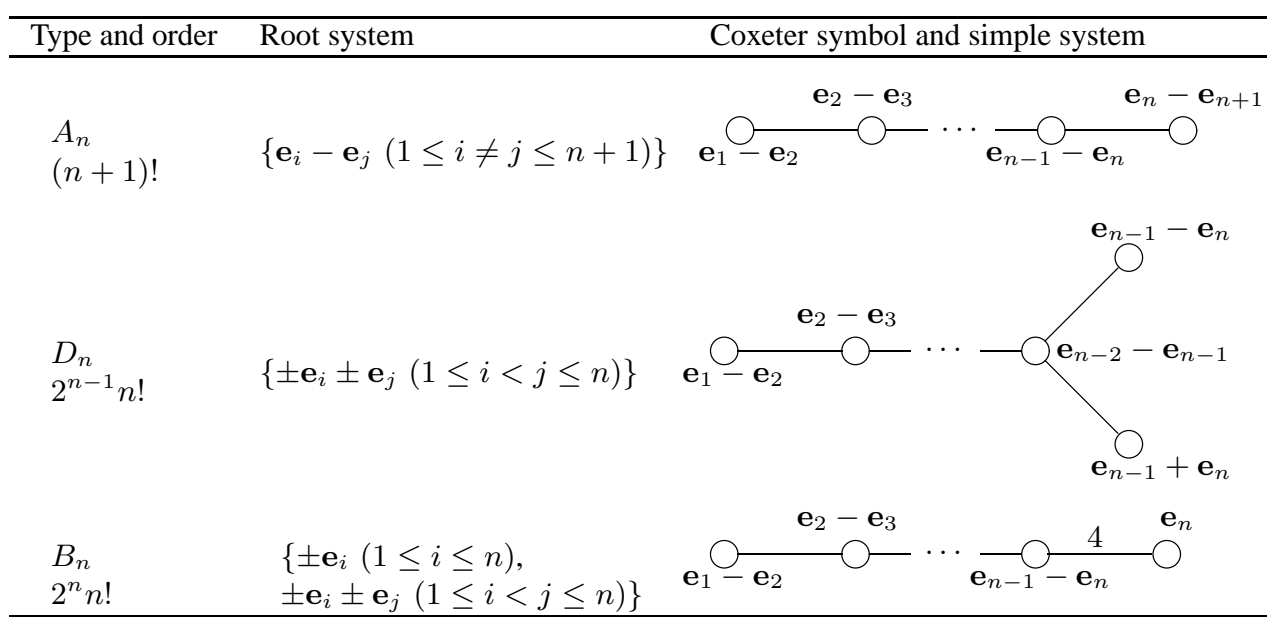

Table 1 Root systems for the classical Weyl groups [17, §2.10]

Consult the root system for $E_{6}$ in Table 2 and make the identifications $x_{1}=s_{\mathbf{e}_{5}-\mathbf{e}_{4}}, x_{2}=$ $s_{\mathbf{e}_{4}-\mathbf{e}_{3}}, x_{3}=s_{\mathbf{e}_{3}-\mathbf{e}_{2}}, x_{4}=s_{\mathbf{e}_{2}-\mathbf{e}_{1}}, x_{5}=s_{\mathbf{v}^{\prime}}$ and $x_{6}=s_{\mathbf{e}_{1}+\mathbf{e}_{2}}$ where $\mathbf{v}^{\prime}=\mathbf{e}_{1}+\mathbf{e}_{8}-\frac{1}{2} \sum_{i=1}^{8} \mathbf{e}_{i}$.

By Theorem [7, $E_{6}$ has a conjugacy class of elements of order three corresponding to the diagram $\coprod_{i=1}^{3} \bigcirc$. A labelling and a colouring is given by,

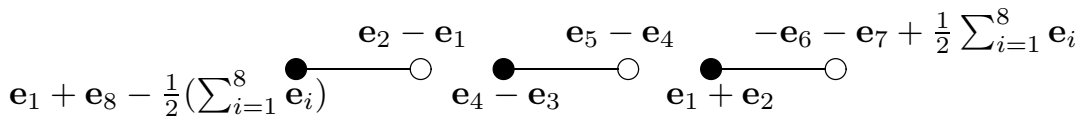

There can be no labelling entirely by simple roots as the diagram is not a subsymbol of the Coxeter symbol for $E_{6}$. Thus, the roots labelling the nodes of the coloured diagram correspond, from left to right, to the reflections $x_{5}, x_{4}, x_{2}, x_{1}, x_{6}$ and $s_{\mathbf{v}}$ for $\mathbf{v}=-\mathbf{e}_{6}-\mathbf{e}_{7}+\frac{1}{2} \sum_{i=1}^{8} \mathbf{e}_{i}$. This last one is not a simple root, but if $w=x_{6} x_{3} x_{2} x_{1} x_{4} x_{3} x_{2} x_{6} x_{3} x_{4}$ (reading from left to right), then in the action of $\Gamma$ on $V, \mathbf{v}$ is sent by $w$ to $\mathbf{v}^{\prime}$ above. When performing these calculations it is helpful to remember that the reflection $s_{\mathbf{e}_{i}-\mathbf{e}_{j}}$ permutes the basis vectors by transposing $\mathbf{e}_{i}$ and $\mathbf{e}_{j}$ while fixing all others. Thus $s_{\mathbf{v}}=w s_{\mathbf{v}^{\prime}} w^{-1}=w x_{5} w^{-1}$ and a representative of the conjugacy class corresponding to this diagram is

$$
x_{5} x_{2} x_{6} x_{4} x_{1} w x_{5} w^{-1}
$$

In [6] a product of this form is read from right to left, so the inverse of "our" element is obtained (as the $s_{\mathbf{v}}$ are involutions). But all the diagrams below have the form

$$
\stackrel{\mathbf{v}_{0}}{\mathrm{O}} \mathbf{v}_{1} \quad \mathbf{v}_{k-1} \quad \mathbf{v}_{k}
$$

so that the subgroup generated by the $s_{\mathbf{v}_{i}}$ is isomorphic to $\mathfrak{S}_{k+1}$, and the two elements obtained are conjugate in $\Gamma$ anyway. Alternatively, although this is far less trivial, any element of a Weyl group is conjugate to its inverse [6, Corollary to Theorem C].

Theorem 5 (types $A$ and $B$ ) The conjugacy classes of prime order in the Weyl groups of types $A_{n}$ and $B_{n}$ correspond to the diagrams: 


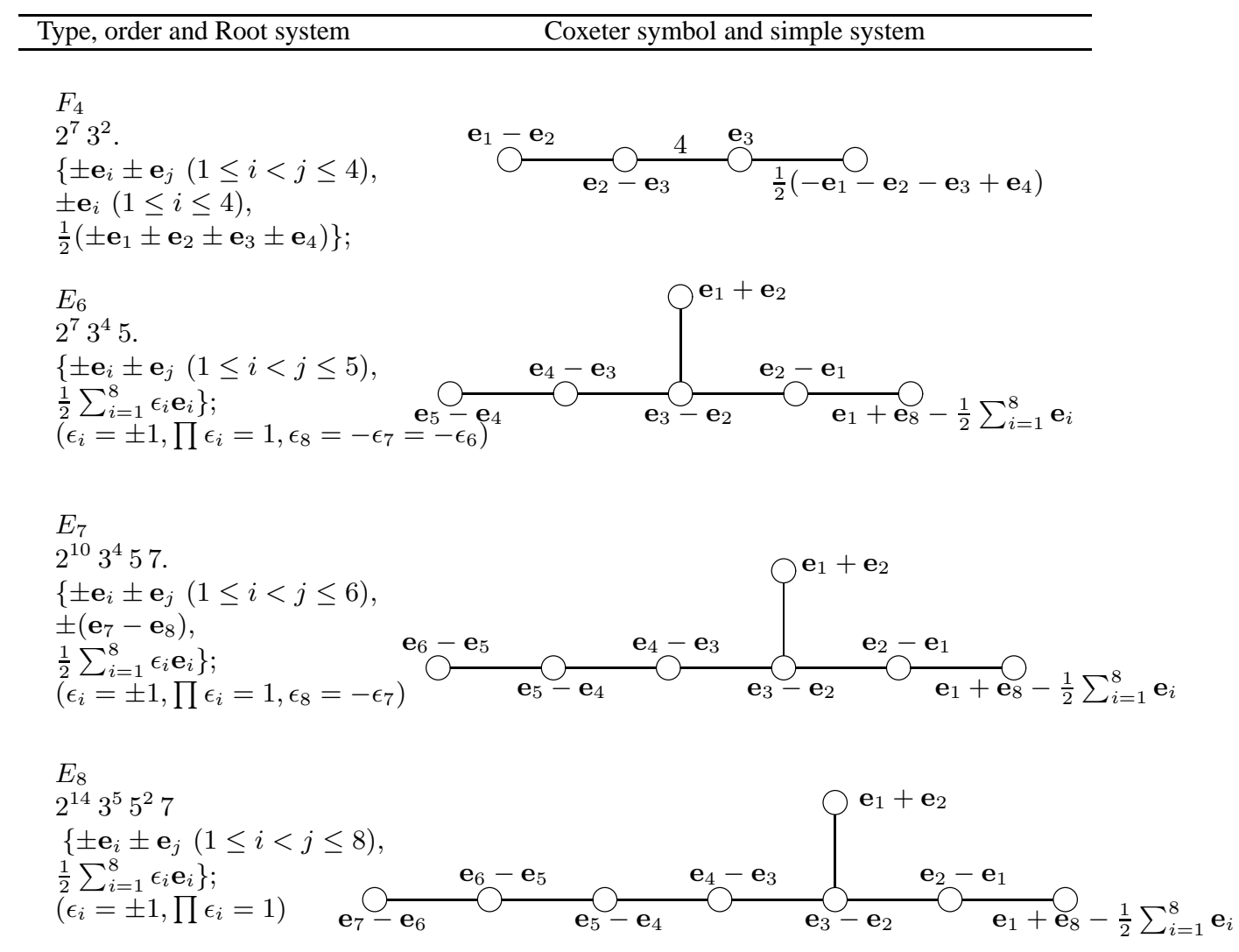

Table 2 Root systems for the exceptional Weyl groups [17, §2.10]

$A_{n}$. order $p \geq 2: \coprod \underbrace{k}_{p-1} \bigcirc \cdots \bigcirc-$ for all $k \geq 1$ with $k p \leq n+1$.
$B_{n}$.

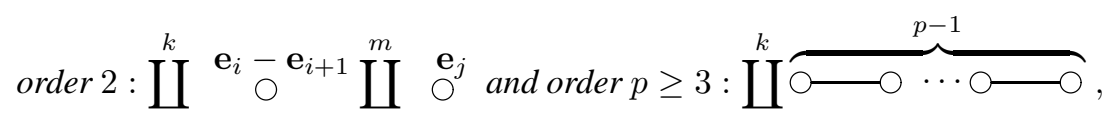

where the order 2 diagrams are for all $k+m>0$ with $2 k+m \leq n$; the order 3 diagrams are for all $k \geq 1$ with $k p \leq n$.

The theorem is Propositions 23 and 24 of [6, §7]. Note that all the unlabelled graphs above can be obtained as subsymbols of the Coxeter symbols for $A_{n}$ and $B_{n}$.

Theorem 6 (type $D)$ The conjugacy classes with prime order in $D_{n}$ correspond to the diagrams: (a) order 2 :

$$
\coprod^{k} \stackrel{\mathbf{e}}{i}^{-\mathbf{e}_{i+1}} \coprod^{m} \mathbf{e}_{j}-\mathbf{e}_{j+1} \mathbf{e}_{j}+\mathbf{e}_{j+1}
$$


for all $k+m>0$ with $2(k+m) \leq n$, except for when $n$ is even and $m=0$, where $\coprod_{i=1}^{n / 2} \bigcirc$ corresponds to two classes with associated diagrams:

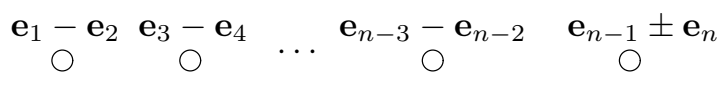

(b) $\operatorname{order} p \geq 3: \coprod \overbrace{\bigcirc-\bigcirc \cdots \bigcirc-\bigcirc}^{p-1}$ for all $k \geq 1$ with $k p \leq n$.

See [6, Proposition 25]. Note the diagram corresponding to two different conjugacy classeshence the need for different labellings. This exception arises from the fact that $D_{n}$ is isomorphic to the subgroup of $B_{n}$ consisting of the even signed permutations of a set of size $n$ : it permutes the basis $\left\{\mathbf{e}_{1}, \ldots, \mathbf{e}_{n}\right\}$ while changing the sign of an even number of them. From the proof of Proposition 25 in [6], if $w_{1}, w_{2}$ are the elements corresponding to the two labellings in part (a) above, and $w w_{1} w^{-1}=w_{2}$, then $w$ must change the sign of an odd number of the basis vectors, so the $w_{i}$ are not conjugate in $D_{n}$ although they are in $B_{n}$.

Theorem 7 (type $E$ ) The conjugacy classes with prime order in the Weyl groups of type $E_{6}, E_{7}$ and $E_{8}$ correspond to the diagrams:

$E_{6}$. (a) order $2: \coprod^{k} \bigcirc,(k=1, \ldots, 4) ;(b)$ order $3: \coprod^{k} \bigcirc,(k=1, \ldots, 3) ;(c)$ order $5:$

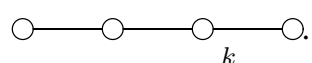

$E_{7}$. (a) order $2: \coprod \bigcirc,(k=1, \ldots, 7)$, except for $k=3,4$, each of which correspond to two classes with associated diagrams:

$$
\begin{array}{cccccc}
\mathbf{e}_{1}-\mathbf{e}_{2} & \mathbf{e}_{3}-\mathbf{e}_{4} & \mathbf{e}_{5} \pm \mathbf{e}_{6} & \mathbf{e}_{1}-\mathbf{e}_{2} & \mathbf{e}_{3}-\mathbf{e}_{4} & \mathbf{e}_{5}-\mathbf{e}_{6} \mathbf{e}_{7}-\mathbf{e}_{8} \\
\bigcirc & \bigcirc & \bigcirc, & \bigcirc & \bigcirc & \bigcirc
\end{array}
$$

(b) $\operatorname{order} 3: \coprod^{k} \bigcirc \bigcirc,(k=1, \ldots, 3) ;(c)$ order $5: \bigcirc-\bigcirc-\bigcirc$

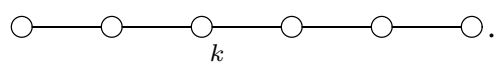

$E_{8} .($ a) order $2: \coprod \bigcirc,(k=1, \ldots, 8)$, except for $k=4$ which corresponds to two classes with associated diagrams:

$$
\begin{array}{cccc}
\mathbf{e}_{1}-\mathbf{e}_{2} & \mathbf{e}_{3}-\mathbf{e}_{4} & \mathbf{e}_{5}-\mathbf{e}_{6} & \mathbf{e}_{7} \pm \mathbf{e}_{8} \\
\bigcirc & \bigcirc & \bigcirc & \bigcirc
\end{array}
$$

(b) $\operatorname{order} 3: \coprod^{k} \bigcirc \mathrm{\bigcirc},(k=1, \ldots, 4)$; (c) order $5: \coprod^{k} \bigcirc \bigcirc-(k=1,2)$;

(d) $\operatorname{order} 7: \mathrm{O}$

Both Theorem 7 and Theorem 8 below arise from a careful examination of the results from [6, §8]. These groups do not have convenient descriptions as the classical groups do. In [6], a collection of non-conjugate elements is formed, and a counting argument gives that the list is complete. As with the groups of type $D_{n}$, there are some pairs of conjugacy classes corresponding to the same (unlabelled) diagram. That the different labellings given in the two theorems yield non-conjugate elements is checked by observing that they fix a different number of roots in the systems from Table 2. 


\begin{tabular}{|c|c|}
\hline Type and order & Coxeter symbol and simple system \\
\hline $\begin{array}{l}H_{3} \\
2^{3} 35 .\end{array}$ & 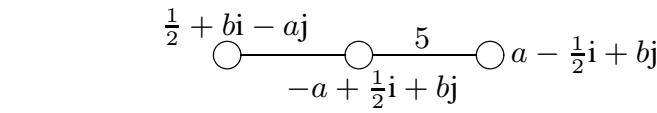 \\
\hline $\begin{array}{l}H_{4} \\
2^{6} 3^{2} 5^{2}\end{array}$ & $\overbrace{-\frac{1}{2}-a \mathrm{i}+b \mathrm{k}} \frac{\frac{1}{2}+b \mathrm{i}-a \mathrm{j}}{-a+\frac{1}{2} \mathrm{i}+b \mathrm{j}}$ \\
\hline
\end{tabular}

Table 3 Root systems for type $H$ non-Weyl groups [17, $\S 2.13]$

Theorem 8 (type $F$ ) The conjugacy classes with prime order in the Weyl group of type $F_{4}$ correspond to the diagrams: (a) order 2 :

$$
\begin{array}{cccc}
\mathbf{e}_{1}-\mathbf{e}_{2} & \mathbf{e}_{1} & \mathbf{e}_{1}-\mathbf{e}_{2} & \mathbf{e}_{3}-\mathbf{e}_{4} \\
\bigcirc, & \bigcirc, & \bigcirc & \bigcirc
\end{array}
$$

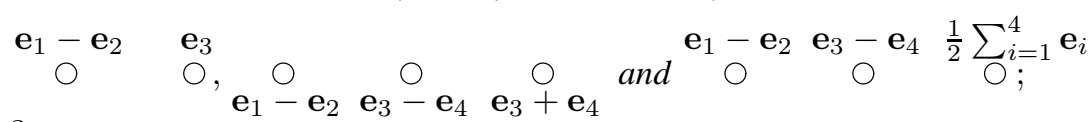

(b) order 3 :

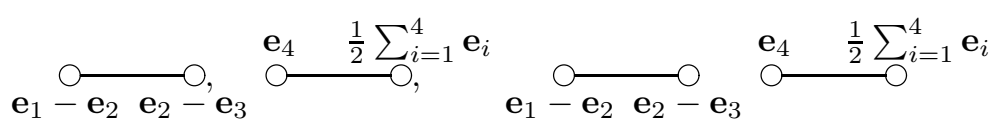

\subsection{The non-Weyl groups}

Identify a 4-dimensional Euclidean space $V$ with the division ring $\mathbf{H}$ of quaternions, so that the bilinear form of $\S 4.1$ becomes $\langle\mathbf{u}, \mathbf{v}\rangle=\frac{1}{2}(\mathbf{u} \overline{\mathbf{v}}+\mathbf{v} \overline{\mathbf{u}})$ where $\overline{\mathbf{v}}=c_{1}-c_{2} \mathbf{i}-c_{3} \mathbf{j}-c_{4} \mathrm{k}$ is the usual quaternionic conjugation. If $\mathbf{v} \in \mathbf{H}$ has norm 1 then the reflection $s_{\mathbf{v}}$ is given by $s_{\mathbf{v}}(\mathbf{u})=-\mathbf{u} \overline{\mathbf{v}} \mathbf{u}$. Let the split extension of $\mathbb{Z}_{2}^{4}$ by $\mathfrak{A}_{4}$ act on $\mathbf{H}$ with the alternating group $\mathfrak{A}_{4}$ permuting coordinates and the $\mathbb{Z}_{2}^{4}$ generated by sign changes. If

$$
a=\frac{1+\sqrt{5}}{4} \text { and } b=\frac{-1+\sqrt{5}}{4}
$$

then the 120 images under this action of $1, \frac{1}{2}(1+\mathrm{i}+\mathrm{j}+\mathrm{k})$ and $a+\frac{1}{2} \mathrm{i}+b \mathrm{j}$ form a root system for $H_{4}$ ([17, §2.13]). The 30 roots orthogonal to $\mathrm{k}$ give a root system for $H_{3}$. Coxeter symbols and simple systems are given in Table 3 .

Proposition 2 (type $H$ ) The conjugacy classes with prime order in the groups of type $H_{3}$ and $\mathrm{H}_{4}$ have representatives:

$H_{3}$. (a) order $2: x_{1}, x_{1} x_{3}$ and $\left(x_{2} x_{1} x_{3}\right)^{5} ;(b)$ order $3: x_{1} x_{2} ;(c)$ order $5: x_{2} x_{3}$ and $\left(x_{2} x_{3}\right)^{2}$.

$H_{4}$. (a) order $2: x_{1}, x_{1} x_{4}, x_{2} x_{1} x_{3} x_{2} x_{1} w^{12} \bar{w} x_{4} x_{3} x_{4}$ and $x_{1} x_{2} x_{1} x_{3} x_{2} x_{1} w^{12} \bar{w} x_{4} x_{3} x_{4} ;(b)$ order $3: x_{1} x_{2}$ and $x_{2} x_{3} ;(c)$ order $5: x_{3} x_{4},\left(x_{3} x_{4}\right)^{2}, x_{3} w^{3} \bar{w} w^{2}, x_{3} w^{9} \bar{w} w^{2}$ and $x_{1} x_{2} x_{1} x_{3} w^{8} \bar{w} x_{4}$ $x_{3} x_{4}$,

where $x_{i}=s_{\mathbf{v}_{i}}$ with $\mathbf{v}_{i}$ the $i$-th simple root from the left in the Coxeter symbol, $\bar{w}=x_{4} x_{3} x_{2}$ and $w$ is the Coxeter element $x_{4} x_{3} x_{2} x_{1}$. 
Proof The 34 conjugacy classes in $H_{4}$, and a representative transformation of $\mathbf{H}$ in each, are given in [14, Table 3]. By observing their effect on 1, i, j, $\mathrm{k}$ the order of these transformations can be computed to give four of order 2, two of order 3 and five of order 5, the others non-prime. Thus it remains to show that the elements stated in the Proposition are non-conjugate. For the order 2 and 3 elements this is most easily done by computing the number of roots fixed each-a tedious but finite task that gives for example in the order 3 case, two roots fixed by $x_{1} x_{2}$ and six by $x_{2} x_{3}$, Three of the order five elements fix no roots, but nevertheless have different traces.

Similar calculations give the $H_{3}$ classes. Alternatively, the group is well known to be isomorphic to $\mathbb{Z}_{2} \times \mathfrak{A}_{5}$, with $\mathfrak{A}_{5}$ the rotations of a dodecahedron, and the center $\mathbb{Z}_{2}$ generated by the antipodal map

Finally we have the, obviously well known,

Proposition 3 (type I) The conjugacy classes with prime order in the dihedral groups,

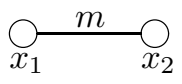

of type I have representatives: for $m=2 k+1, x_{1}$ and $\left(x_{1} x_{2}\right)^{l}$; and for $m=2 k, x_{1}, x_{2}$ and $\left(x_{1} x_{2}\right)^{l}$; for all $l \leq k$ with $\mathrm{m} / \operatorname{gcd}(m, l)$ is prime.

\section{Volume}

Let $\Pi$ be a group acting properly, freely, cofinitely by isometries on $\mathbb{H}^{n}$ and let $M=\mathbb{H}^{n} / \Pi$. If $n$ is even then the Gauss-Bonnet (-Hirzebruch) formula [28, 13, 15, 16], gives

$$
\operatorname{vol}(M)=\kappa_{n} \chi_{\text {top }}(M)
$$

with $\chi_{\text {top }}(M)$ the Euler characteristic of $M$ and $\kappa_{n}=2^{n}(n !)^{-1}(-\pi)^{n / 2}(n / 2)$ ! As $\Pi$ is of finite homological type and torsion free the Euler characteristic, $\chi(\Pi)=\sum_{i}(-1)^{i} \operatorname{rank}_{\mathbb{Z}} H_{i}(\Pi)$ is defined [3, $\S \mathrm{IX} .6]$, and since $M$ is a $K(\Pi, 1)$ space ( $\mathbb{H}^{n}$ being contractible) the homologies $H_{*}(M) \cong H_{*}(\Pi)$, so $\chi_{\text {top }}(M)=\chi(\Pi)$.

Now suppose $\Pi$ arises via a torsion free transitive $\mathbb{C} \Gamma$-module $U$ as in $\S 3$, with $\Gamma$ a hyperbolic Coxeter group and $\operatorname{dim} U=m$ (which is thus the index in $\Gamma$ of $\Pi$ ). Then $\chi(\Gamma)$ is defined and $\chi(\Pi)=m \chi(\Gamma)$. But calculating the Euler characteristic of Coxeter groups turns out to be a simple task:

Theorem 9 Let $(\Gamma, S)$ be a Coxeter group. For any $\sigma \in \mathscr{F}$, let $|\sigma|$ be the order of this group. Then,

$$
\chi(\Gamma)=\sum_{\substack{\sigma_{0}<\cdots<\sigma_{k} \\ \sigma_{i} \in \mathscr{F}}} \frac{(-1)^{k}}{\left|\sigma_{0}\right|},
$$

where the sum is over all chains $\sigma_{0}<\cdots<\sigma_{k}$ with $\sigma_{i} \in \mathscr{F}$.

A similar result appears in [7] for the Euler characteristic defined in [8]. 
Proof Let $\Sigma$ be the affine $\Gamma$-complex from $\S 2$, metrised as there to be a CAT $(0)$ space. For each $k$-cell $\sigma=\left(\sigma_{0}<\sigma_{1}<\cdots<\sigma_{k}\right)$ let $\Gamma_{\sigma}$ be the isotropy group of $\sigma$ and $Y$ the $k$-cells $\sigma_{0}<\sigma_{1}<\cdots<\sigma_{k}$ where each $\sigma_{i} \in \mathscr{F}$. Then $Y$ is a set of representatives of the cells of $\Sigma$ modulo the $\Gamma$-action. Moreover, $\Sigma$ is contractible (as indeed is any CAT(0) space) so by [ $\beta$, Proposition 7.3( $\left.\mathrm{e}^{\prime}\right)$,

$$
\chi(\Gamma)=\chi_{\Gamma}(\Sigma)=\sum_{\sigma \in Y}(-1)^{\operatorname{dim} \sigma} \chi\left(\Gamma_{\sigma}\right),
$$

with the right hand side the $\Gamma$-equivariant Euler characteristic of $\Sigma$. If $\sigma=\left(\sigma_{0}<\cdots<\sigma_{k}\right) \in Y$ then $\Gamma_{\sigma}=\sigma_{0}$ and $\chi\left(\Gamma_{\sigma}\right)=1 /\left|\Gamma_{\sigma}\right|=1 /\left|\sigma_{0}\right|$ as these group are finite.

If $\Gamma$ is a Coxeter symbol and $\Delta$ a subsymbol, let $\Gamma \backslash \Delta$ be the subsymbol obtained by removing from $\Gamma$ the vertices of $\Delta$ and their incident edges. The following lemma is then sometimes useful.

Lemma 2 Let $\Gamma$ be a Coxeter symbol and $\Psi$ a subsymbol. Let $\Sigma_{\Psi}$ be the sum (1) restricted to those chains $\sigma_{0}<\cdots<\sigma_{k}$ with $\Psi<\sigma_{0}$. Then,

$$
\Sigma_{\Psi}=\sum_{\Delta \in \mathscr{P}(\Psi)}(-1)^{v(\Delta)} \chi(\Gamma \backslash \Delta),
$$

where $\mathscr{P}(\Psi)$ is the set of all subsymbols of $\Psi$, and $v(\Delta)$ the number of nodes of $\Delta$.

Proof Apply the inclusion-exclusion principle as in [29, §2.1]

If $\Psi$ is the symbol for an infinite Coxeter group, then $\sum_{\Psi}=0$, a sum over an empty set, so in particular if $\Gamma$ is infinite,

$$
\sum_{\Delta \in \mathscr{P}(\Gamma)}(-1)^{v(\Delta)} \chi(\Gamma \backslash \Delta)=0,
$$

which is Serre's closed form for the Euler characteristic [27, Proposition 16]

For $n$ odd, the Euler characteristic of an $n$-dimensional hyperbolic Coxeter group is zero, and there is in general no simple method for computing volumes. Of course, $\operatorname{vol}\left(\mathbb{H}^{n} / \Pi\right)=m \operatorname{vol}(P)$ where $\Pi$ is an index $m$ subgroup of Coxeter group $\Gamma$ with fundamental polytope $P$. For certain $\Gamma$ 24] the volumes of such $P$ has been determined.

\section{Examples}

We give three manifolds as examples of the construction, one in each $\mathbb{H}^{n}$ for $n=4,5,6$. We have let volume be our guiding principle, so that the three are either the smallest possible $(n=4)$ or would appear to be the smallest known $(n=5,6)$. As mentioned in the Introduction, a large family of 4-manifolds with $\chi=1$ have recently been constructed by Ratcliffe and Tschantz [23] using a computer.

We first explain the notation. In each case we have a Coxeter group $\Gamma$ acting on a collection of finite sets $\Omega_{i}$, so that if $U_{i}$ is the $\mathbb{C}$-vector space with basis $\Omega_{i}$, then $\otimes U_{i}$ is a torsion free $\mathbb{C} \Gamma$-module.

The actions of $\Gamma$ on the $\Omega_{i}$ are depicted using two notations. If $\Omega_{i}$ has solid black nodes (as in 6.1, 6.2 diagrams (1)-(3) and 6.3 diagrams (1)-(2)) then generator $x_{j}$ of $\Gamma$ acts by swapping 
two nodes if they are connected by an edge labelled according to the scheme: — for $x_{1}$; for $x_{2} ; \multimap \odot$ for $x_{3} ; \multimap-\circ$ for $x_{4} ; \multimap$ for $x_{5} ; \multimap$ for $x_{6}$ and —— for $x_{7}$ (or even combinations that can be interpreted unambiguously like $=0=$ for an $x_{2}$ and $x_{3}$ edge). Nodes not so connected are fixed by the corresponding $x_{j}$.

The other notation, used in 6.2 diagram (4) and 6.3 diagrams (3)-(4), is convenient when the action of $\Gamma$ is imprimitive and we want to compress rather a lot of information (and so the resulting diagrams require a certain amount of decoding). Recall that if a group $\Gamma$ acts on a set $\Omega$, then this action is imprimitive if $\Omega=\bigcup \Omega_{i}$, with the $\Omega_{i}$ disjoint, and $\Omega_{i}^{\gamma}=\Omega_{j}$ for all $i$ and $\gamma \in \Gamma$ (ie: the action on $\Omega$ induces an action on the $\Omega_{i}$ ). The $\Omega_{i}$ are the blocks of imprimitivity, all of the same cardinality if the action is transitive. The numbered vertices in 6.2(4) and 6.3(3)(4) are the blocks of imprimitivity of an imprimitive $\Gamma$-action; the action on the blocks is depicted as above, so that for instance, blocks 7 and 9 in 6.3(3) are interchanged by generator $x_{3}$.

To recover the action on the original set $\Omega$, suppose that the block $\Omega_{i}=\left\{m_{i 1}, m_{i 2}, \ldots, m_{i k}\right\}$. For $i<j$, we use the notation (i) ${ }^{\sigma}$ (j) to mean $m_{i l}$ goes to $m_{j \sigma(l)}$ under the action of the generator labelling the edge (which we've left off here for clarity). Edges unlabelled by a permutation in this way correspond to $\sigma$ being the identity. If the block $\Omega_{i}$ is fixed by $x_{j}$, then the action of $x_{j}$ on the $m_{i l}$ is given with each diagram.

6.1 A 4-manifold with $\chi=1$.

Let $\Gamma$ be the Coxeter group with symbol on the left,

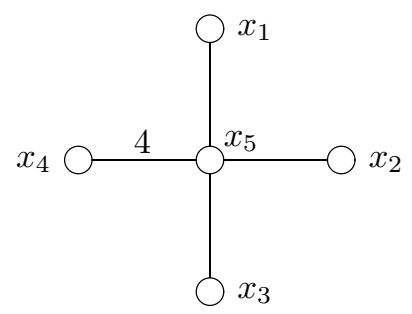

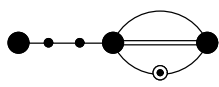

(1)

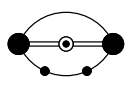

(3)

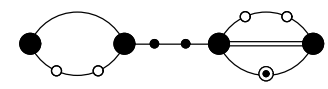

(2)

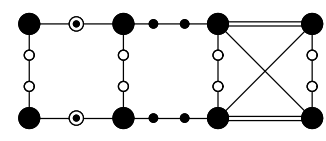

(4)

which appears in $[17, \S 6.9]$, although it is easily checked that the Gram matrix $G(\Gamma)$ has signature $(1,4)$ and that the poset $\overline{\mathscr{F}}$ is isomorphic to the poset of a combinatorial 4 -simplex. Thus, by Theorems 1 2 $2, \Gamma$ acts cofinitely on $\mathbb{H}^{4}$ with fundamental region a cusped 4 -simplex.

From $\mathscr{F}$ and Theorem 9 we have

$$
\chi(\Gamma)=1 / \mathscr{L}(\Gamma)
$$

where $\mathscr{L}(\Gamma)=2^{6} 3$, the lowest common multiple of the order of the elements of $\mathscr{F}$, is found using Tables 1 3 . There are 11 finite subgroups generated by maximal $S^{\prime} \subseteq S$ : a $D_{4}$, three $B_{3}$ 's, three $A_{3}$ 's, three $A_{1} \times A_{1} \times A_{1}$ 's and a $A_{1} \times A_{1} \times A_{1} \times A_{1}$, and conjugacy class representatives in these groups can be listed using the results of $\S 4$. For example, $x_{1}, x_{2}, x_{3}$ and $x_{5}$ generate the Weyl group of type $D_{4}$. Consult the root system in Table 1 and make the identifications $x_{2}=s_{\mathbf{e}_{1}-\mathbf{e}_{2}}, x_{5}=s_{\mathbf{e}_{2}-\mathbf{e}_{3}}, x_{1}=s_{\mathbf{e}_{3}-\mathbf{e}_{4}}$ and $x_{3}=s_{\mathbf{e}_{3}+\mathbf{e}_{4}}$. By Theorem 6 the order two torsion is corressponds to the diagrams,

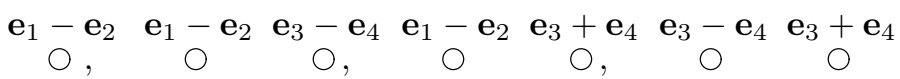




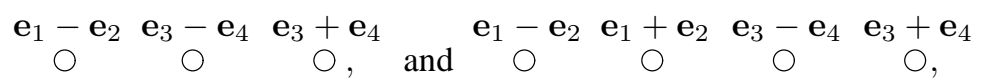

with resulting representatives $x_{2}, x_{1} x_{2}, x_{2} x_{3}, x_{1} x_{3}, x_{1} x_{2} x_{3}$ and $x_{2} s_{\mathbf{e}_{1}+\mathbf{e}_{2}} x_{1} x_{3}$, where $s_{\mathbf{e}_{1}+\mathbf{e}_{2}}=$ $w x_{3} w^{-1}$ with $w=x_{5} x_{1} x_{2} x_{5}$; the order three torsion consists of the single class

$$
\mathbf{e}_{1}-\mathbf{e}_{2} \mathbf{e}_{2}-\mathbf{e}_{3}
$$

with corresponding representative $x_{2} x_{5}$. Perform this process for the 10 other groups.

The diagrams labelled $(i)$ above give actions of the $x_{j}$ on sets $\Omega_{i}$ using the notation from the beginning of the section. By checking that each relator word $\left(s_{\alpha} s_{\beta}\right)^{m_{\alpha \beta}}$ acts as the identity on $\Omega_{i}$, it can be seen that the diagrams give actions of $\Gamma$ on $\Omega_{i}$. Let $U_{i}$ be the resulting $\mathbb{C} \Gamma$-module, transitive in each case as the diagrams are connected.

One now checks, using Proposition 1, that each representative of torsion in $\Gamma$ is avoided by at least one of the $U_{i}$ : for instance, those listed above are avoided by $U_{3}, U_{2}, U_{4}, U_{2}, U_{1}, U_{4}$ and $U_{1}$ respectively. Thus $U=\otimes U_{i}$ is torsion free. The index in $\Gamma$ of the corresponding subgroups $\Pi$ is at most $\prod\left|\Omega_{i}\right|=2^{6} 3=\mathscr{L}(\Gamma)$. On the otherhand, the index of any torsion free subgroup must be a multiple of $\mathscr{L}(\Gamma)$, hence the index is $\mathscr{L}(\Gamma)$. We thus obtain a (non-compact) hyperbolic 4-manifold $M=\mathbb{H}^{4} / \Pi$ with $\chi(M)=\chi(\Pi)=\mathscr{L}(\Gamma) \times \chi(\Gamma)=1$.

6.2 A 5-manifold with volume $\leq 14 \zeta(3)$

The Coxeter symbol,

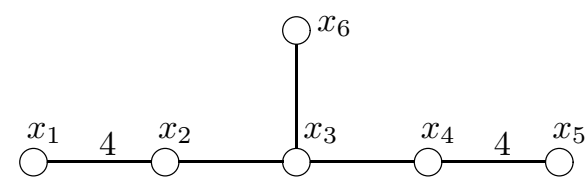

corresponds to a 5-dimensional hyperbolic group acting cofinitely on $\mathbb{H}^{5}$ with fundamental region a cusped 5-simplex ([17, §6.9] or Theorems 1-2 The four diagrams,

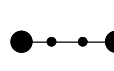

(1)

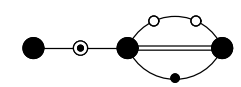

(2)

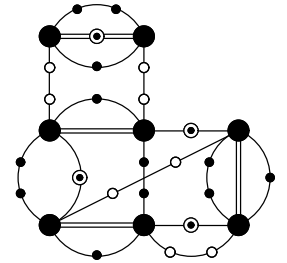

(3)

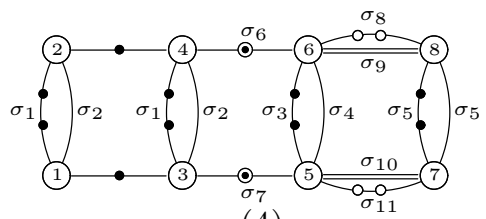

(4)

give $\mathbb{C} \Gamma$-modules $U_{i}$ as in $\S 6.1$. In diagram (4), each block of imprimitivity has size eight, giving a $\Gamma$-action on $\Omega_{4}$ with $\left|\Omega_{4}\right|=64$. If $x_{j}$ fixes block $i$, the action of $x_{j}$ on the eight points in the

\begin{tabular}{|c|c|c|}
\hline $\mathbf{1 : 2 ( 2 , 4 ) ( 5 , 7 ) , 3 ( 3 , 5 ) ( 4 , 6 ) , 4 ( 2 , 5 ) ( 4 , 7 )}$ & $4: 2(1,3)(6,8), 4(2,4)(5,7)$ & $\mathbf{7 : 3 ( 3 , 6 ) ( 4 , 7 ) , 6 ( 5 , 7 ) ( 6 , 8 )}$ \\
\hline $\mathbf{2}: 2(1,3)(6,8), 3(3,4)(5,6), 4(2,4)(5,7)$ & $\mathbf{5 : 6 ( 4 , 6 ) ( 5 , 7 )}$ & 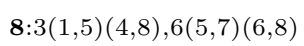 \\
\hline $3: 2(2,4)(5,7), 4(2,5)(4,7)$ & $6: 6(3,4)(7,8)$ & \\
\hline
\end{tabular}
block is as a permutation $\sigma$ given by, 
using the form, $\mathbf{i}: j \sigma, \ldots$ The permutations $\sigma_{i}$ in the diagram are given by,

\begin{tabular}{l|l|l}
\hline$\sigma_{1}=(1,2)(3,5,6,4)(7,8)$ & $\sigma_{5}=(1,3)(5,6)(7,8)$ & $\sigma_{9}=(2,3,5)(4,7,6)$ \\
$\sigma_{2}=(4,5)$ & $\sigma_{6}=(5,7)(6,8)$ & $\sigma_{10}=(2,3)(4,5,6,7,8)$ \\
$\sigma_{3}=(1,2)(3,5)(4,7)(6,8)$ & $\sigma_{7}=(3,4,5,6,7,8)$ & $\sigma_{11}=(1,2,4,5,6,7,8,3)$ \\
$\sigma_{4}=(3,6,4)(5,7,8)$ & $\sigma_{8}=(1,2,4,8,7,5)(3,6)$ & \\
\hline
\end{tabular}

Listing the torsion in $\Gamma$ using the results of $\S 4$, one can check that the $U_{i}$ collectively avoid all torsion, so that $U=\otimes U_{i}$ is a torsion free $\mathbb{C} \Gamma$-module. There is no Euler characteristic in 5 -dimensions, but according to [24], a cusped 5-simplex fundamental region for $\Gamma$ has volume $7 \zeta(3) / 2^{9} 3$, with $\zeta$ the Riemann-zeta function. The index in $\Gamma$ of the resulting $\Pi$ is $\leq \prod\left|\Omega_{i}\right|=$ $2^{2} \mathscr{L}(\Gamma)$, thus $\frac{7}{2} \zeta(3) \leq \operatorname{vol}(M) \leq 14 \zeta(3)$.

6.3 A 6-manifold with $|\chi| \leq 16$

The Coxeter symbol,

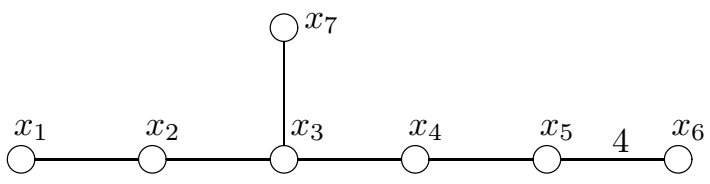

is, as the others, that of a $\Gamma$ acting cofinitely on $\mathbb{H}^{6}$ with fundamental region a cusped 6 -simplex [17, §6.9]. The two diagrams,

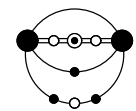

(1)

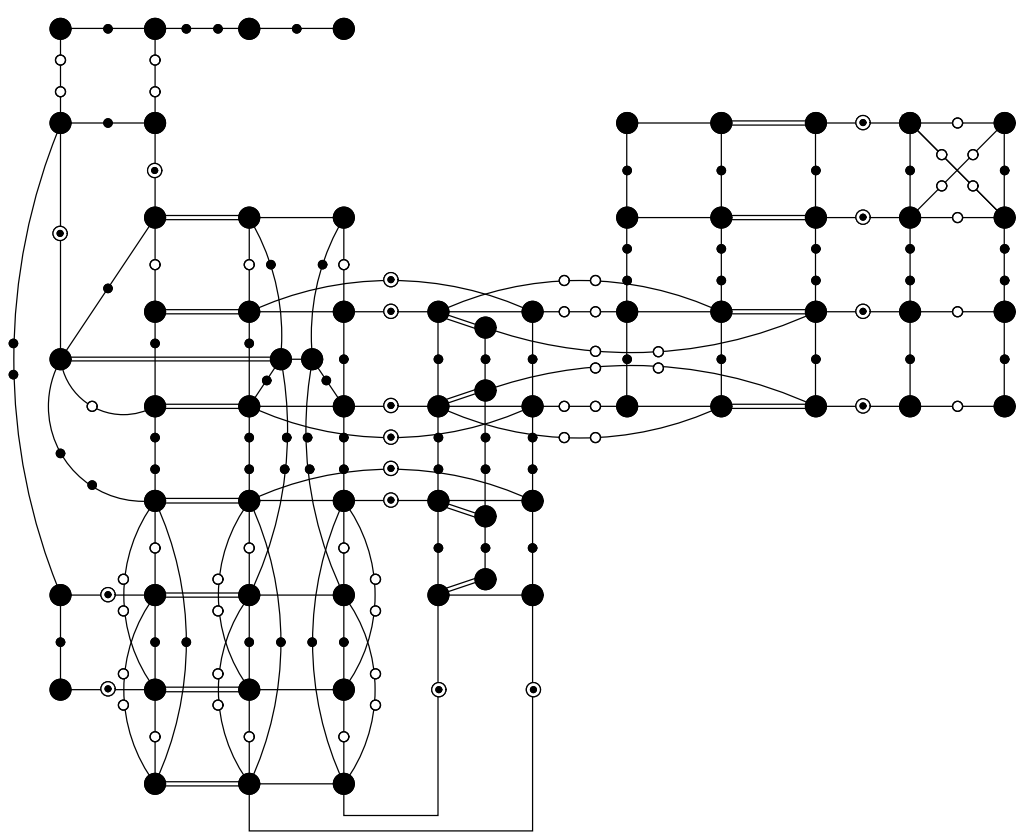

(2) 
give primitive $\Gamma$-actions on 2 and 64 points. Diagram (3) below depicts an action with 27 blocks of imprimitivity, each of size six:

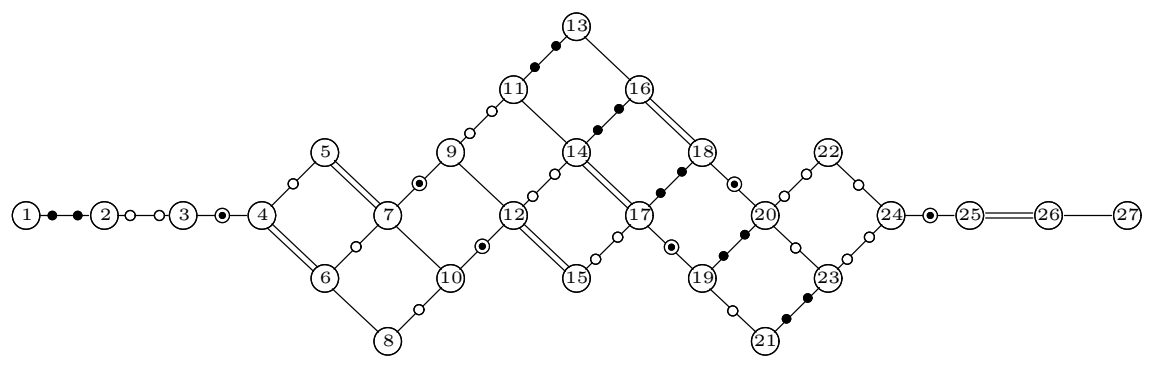

If $x_{j}$ fixes block $\Omega_{i}$ then the action on the six points of $\Omega_{i}$ is a product of three disjoint 2-cycles. Partially order the elements of $\mathfrak{S}_{6}$ by $\sigma<\tau$ if and only if $\sigma(i)<\tau(i)$, where $i$ is the smallest element of $\{1, \ldots, 6\}$ on which $\sigma$ and $\tau$ differ. Restrict this order to the permutations of type three disjoint 2-cycles. The $x_{j}$ action on $\Omega_{i}$ is then as,

\begin{tabular}{|c|c|c|}
\hline $\mathbf{1}: 1(3) 2(4) 3(8) 4(1) 7(1)$ & 10:2(3)4(8)5(1) & 19:1(1)2(8)4(3) \\
\hline 2:1(3)2(4)3(8)7(1) & 11:2(1)3(8)7(4) & 20:1(1)2(8) \\
\hline 3:1(3)2(4)5(1)7(1) & 12:5(1)7(4) & 21:1(1)2(8)3(4)4(3) \\
\hline $4: 1(3) 4(8) 5(1)$ & 13:2(1)3(8)4(1)7(4) & 22:1(1)2(8)5(3) \\
\hline $\mathbf{5 : 1 ( 3 ) 3 ( 1 ) 4 ( 8 ) 5 ( 1 )}$ & 14:3(8)7(4) & 23:1(1)2(8)3(4) \\
\hline 6:3(4)4(8)5(1) & 15:1(1)3(3)5(1)7(4) & 24:1(1)2(8)5(3) \\
\hline $\mathbf{7 : 4 ( 8 ) 5 ( 1 )}$ & 16:3(8)4(1)7(4) & 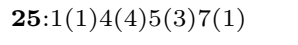 \\
\hline $8: 2(3) 3(4) 4(8) 5(1)$ & $\mathbf{1 7}: 1(1) 7(4)$ & 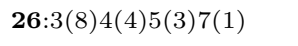 \\
\hline 9:2(1)5(1)7(4) & 18:1(1)4(1)7(4) & $\mathbf{2 7}: 2(1) 3(8) 4(4) 5(3) 7(1)$ \\
\hline
\end{tabular}

using the form, $\mathbf{i}: j(k) \ldots$, where $x_{j}$ acts as the $k$-th permutation of the form three disjoint 2-cycles.

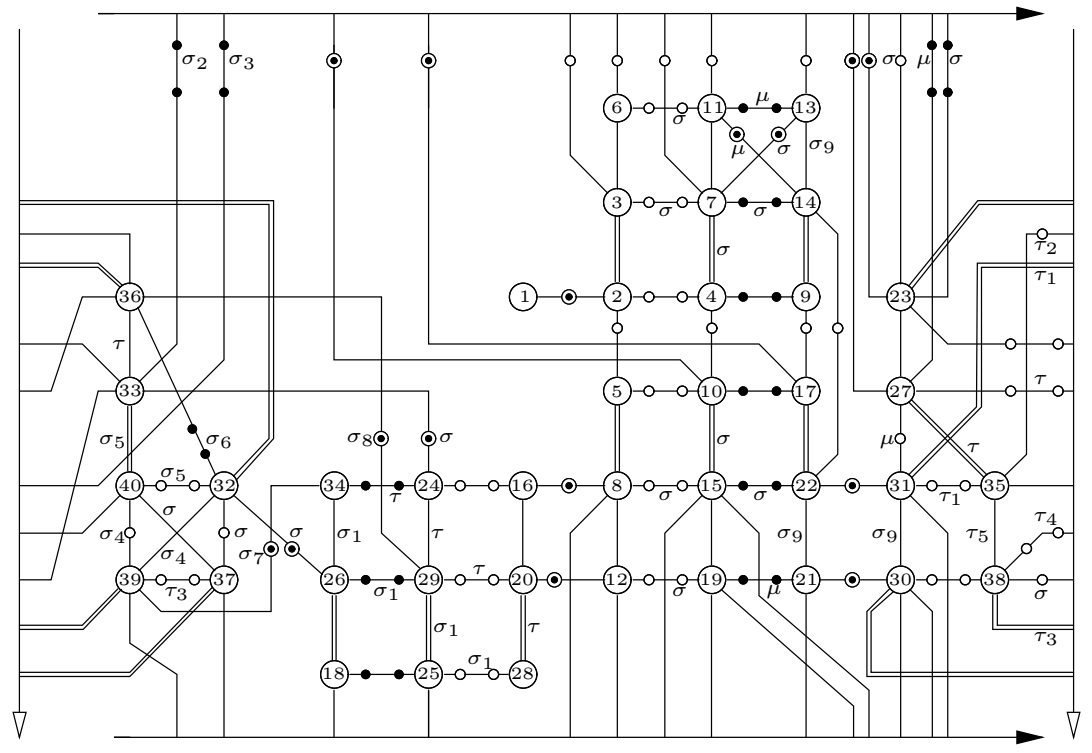


Finally, diagram (4) above gives a $\Gamma$-action on 40 blocks of imprimitivity, each of size eight, and drawn on the torus. Those generators fixing blocks act on them as the product of four disjoint 2 -cycles. Taking the order above on $\mathfrak{S}_{8}$ and restricting it to just the permutations of this type, these actions are,

\begin{tabular}{|c|c|c|c|}
\hline $\begin{array}{l}\mathbf{1}: 1(8) 2(8) 4(24) \\
\quad 5(24) 6(42) 7(59)\end{array}$ & 11:2(8)6(24) & $\mathbf{2 1}: 2(24) 4(8) 6(42)$ & 31:6(42) \\
\hline 2:1(8)5(24)6(42) & 12:2(8)5(24)6(42) & 22:4(24)6(42) & 32:6(24) \\
\hline 3:3(8)5(24)6(42) & 13:2(24)4(8)6(42) & 23:6(24) & 33:6(24) \\
\hline $4: 1(8) 3(24) 6(42)$ & $\mathbf{1 4}: 4(24) 6(42)$ & $\mathbf{2 4 : 2 ( 5 9 ) 6 ( 4 2 ) 7 ( 8 )}$ & 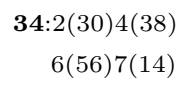 \\
\hline 5:1(8)3(59)5(24)6(42) & 15:6(24) & 25:1(8)6(39)7(24) & $\mathbf{3 5}: 3(14) 6(38)$ \\
\hline 6:2(8)3(8)5(24)6(42) & 16:2(59)5(24)6(42)7(8) & 26:4(59)6(42)7(24) & 36:6(38) \\
\hline $\mathbf{7 : 6 ( 2 4 )}$ & $\mathbf{1 7}: 1(8) 4(24) 6(42)$ & $\mathbf{2 7}: 6(24)$ & $\mathbf{3 7}: 3(24) 6(42)$ \\
\hline $8: 5(24) 6(42)$ & 18:1(8)4(59)6(39)7(24) & $\begin{array}{l}\mathbf{2 8}: 1(30) 3(14) 5(38) \\
\quad 6(56) 7(14)\end{array}$ & $\mathbf{3 8 : 3 ( 8 ) 6 ( 4 2 )}$ \\
\hline 9:1(8)3(24)4(24)6(42) & 19:2(8)6(24) & 29:6(56)7(14) & 39:6(38) \\
\hline 10:1(8)6(42) & 20:5(24)6(42)7(8) & 30:6(42) & $40: 3(42) 6(24)$ \\
\hline
\end{tabular}

using the same notation as for diagram (3). The permutations given in the diagram are,

\begin{tabular}{l|l|l|l}
\hline$\sigma=(3,4)(6,7)$ & $\sigma_{5}=(4,5)(7,8)$ & $\tau=(3,4,5)$ & $\tau_{1}=(2,3)(6,8)$ \\
$\sigma_{1}=(2,3)(4,5)(6,8,7)$ & $\sigma_{6}=(2,3,4)(6,7,8)$ & $\mu=(2,3,4)(6,8,7)$ & $\tau_{4}=(2,4,3,5)(7,8)$ \\
$\sigma_{2}=(2,3,4,5)(6,7)$ & $\sigma_{7}=(2,3)(4,5)$ & $\tau_{1}=(2,3)(6,8)$ & \\
$\sigma_{3}=(2,5,3)(6,7,8)$ & $\sigma_{8}=(4,5)(6,7)$ & $\tau_{2}=(3,5)(7,8)$ & \\
$\sigma_{4}=(3,4)(7,8)$ & $\sigma_{9}=(2,3)(7,8)$ & $\tau_{3}=(3,5)(6,7)$ & \\
\hline
\end{tabular}

As per usual, let $U=\otimes U_{i}$, having checked that the $U_{i}$ collectively avoid the torsion in $\Gamma$. From $\mathscr{F}$ and Theorem 9 we have $\chi(\Gamma)=-1 / \mathscr{L}(\Gamma)$, while the index in $\Gamma$ of the resulting $\Pi$ is $\leq \prod\left|\Omega_{i}\right|=2^{4} \mathscr{L}(\Gamma)$, thus $|\chi| \leq 16$.

\section{References}

1. N Bourbaki. Groupes et algébres de Lie. Chapters 4-6, Hermann, Paris 1968; Masson, Paris 1981.

2. M Bridson and A Haefliger. Metric Spaces of Non-positive Curvature. Grundlehren der mathematischen Wissenschaften, Springer, 1999.

3. K Brown. Cohomology of Groups. Graduate Texts in Math 87, Springer, 1982.

4. V O Bugaenko. The automorphism groups of unimodular hyperbolic quadratic forms over $\mathbb{Z}[(1+$ $\sqrt{5}) / 2]$. Moskov. Univ. Vestnik Ser. 1 Mat. Mekh., 5 (1984), 6-12.

5. B Brink and R Howlett. A finitness property and an automatic structure for Coxeter groups. Math. Ann. 296 (1993), 179-190.

6. R W Carter. Conjugacy classes in the Weyl group. Compositio Mathematica, 25:1 (1972), 1-59.

7. I M Chiswell. The Euler characteristic of graph products and Coxeter groups. in Discrete Groups and Geometry, W J Harvey and Colin Maclachlan (Editors), London Maths. Soc. Lect. Notes, 173 (1992), 36-46.

8. I M Chiswell. Euler characteristics of groups. Math. Z., 147 (1976), 1-11.

9. M W Davis and G Moussong. Notes on nonpositively curved polyhedra. in Low Dimensional Topology, Budapest (Hungary) 1998, Bolyai Society Mathematical Studies, 8, 11-94, 1999. 
10. M W Davis. A hyperbolic 4-manifold. Proc. Amer. Math. Soc., 93 (1985), 325-328.

11. A L Edmonds, J H Ewing and R S Kulkarni. Torsion free subgroups of Fuchsian groups and tessellations of surfaces. Invent. Math. 69 (1982), 331-346.

12. B Everitt and C Maclachlan. Constructing hyperbolic manifolds. in Computational and Geometric Aspects of Modern Algebra, Michael Atkinson et al.(Editors), London Maths. Soc. Lect. Notes, 275 (2000), 78-86.

13. M Gromov. Volume and bounded cohomology. Publ. Math. Inst. Hautes Études Sci., 76 (1982), $165-246$

14. L Grove. The characters of the hecatonicosahedroidal group. J. Reine Angew. Math. 265 (1974), $160-169$.

15. F Hirzebruch. Characteristic numbers of homogenous domains, in Seminars on Analytic Functions Vol. II, Institute for Advanced Studies, Princeton, 92-104, 1957.

16. F Hirzebruch. Topological methods in Algebraic Geometry, Grundlehren Math. Wiss., 131, Springer, New York, 1966.

17. J F Humphreys. Reflection groups and Coxeter groups, Cambridge studies in advanced mathematics, CUP, 1990.

18. K N Jones and A W Reid. Minimal index torsion-free subgroups of Kleinian groups. Math. Ann. 310 (1998), 235-250.

19. G A Margulis. Isometry of closed manifolds of constant negative curvature with the same fundamental group. Soviet Math. Dokl. 11 (1970) 722-723.

20. G D Mostow. Strong rigidity of locally symmetric spaces. Ann. of Math. Studies 78, Princeton, (1973).

21. G Moussong. Hyperbolic Coxeter Groups. PhD thesis, University of Ohio, 1988.

22. G Prasad. Strong rigidity of $\mathbb{Q}$-rank 1 lattices. Invent. Math. 21 (1973) 255-286.

23. J Ratcliffe and S Tschantz. The volume spectrum of hyperbolic 4-manifolds. Experiment. Math. 9 (2000), no. 1, 101-125.

24. N Johnson, R Kellerhals, J Ratcliffe and S Tschantz. The size of a hyperbolic Coxeter simplex. Transform. Groups 4 (1999), no. 4, 329-353.

25. J Ratcliffe and S Tschantz. Volumes of integral congruence hyperbolic manifolds. J. Reine Angew. Math. 488 (1997), 55-78.

26. A Selberg. On discontinuous groups in higher dimensional symmetric spaces, in Contributions to Function Theory K Chandrasekharan editor, Tata Inst. of Fund. Research, Bombay (1960), 147-164.

27. J-P Serre. Cohomologie des groupes discrets in, Prospects in Mathematics, Annals of Mathematical Studies 70, 77-169, Princeton University Press, 1971.

28. M Spivak. A Comprehensive Introduction to Differential Geometry, 2nd eition, Publish or Perish, Wilmington, 1979.

29. Richard P. Stanley. Enumerative Combinatorics, Cambridge Studies in Advanced Mathematics, CUP, 1997.

30. E B Vinberg. Hyperbolic Reflection Groups. Uspekhi Mat. Nauk 40:1 (1985), 29-66, = Russian Math. Surveys 40:1 (1985), 31-75.

31. E B Vinberg. Discrete groups in Lobachevskii spaces generated by reflections Math. Sb. 72 (1967), 471-488. = Math USSR-Sb. 1 (1967), 429-444. 